\title{
Autoinflammatory diseases in pediatrics
}

\author{
Silvia M. Meiorin, M.D. ${ }^{a}$, Graciela Espada, M.D. ${ }^{a}$ and Carlos Rosè, M.D. ${ }^{b}$
}

\begin{abstract}
Monogenic autoinflammatory syndromes are caused by mutations in protein-coding genes that have a pivotal role in the regulation of the inflammatory response. Due to their genetic nature, most of these syndromes usually begin during childhood. They are clinically characterized by recurrent episodes of systemic inflammation (fever with different clinical manifestations, such as skin rash, serositis or arthritis) associated with elevation of acute phase reactants. During symptomfree intervals, patients achieve clinical wellbeing and normalize inflammatory parameters. Amyloidosis is a serious long-term complication. In this update we will discuss the clinical presentation and therapeutic strategies for these diseases in pediatrics.

Key words: hereditary autoinflammatory diseases, hereditary periodic fever syndromes, inflammasome.
\end{abstract}

http:/ /dx.doi.org/10.5546/aap.2013.237

Autoinflammatory syndromes include diseases secondary to mutations in protein-coding genes that play a pivotal role in the regulation of the inflammatory response. Given their genetic nature, most syndromes have an early onset, and in some cases even within the first hours of life, while they rarely begin in adulthood. Since they are not frequent and have been recently identified, these syndromes often result in a delayed diagnosis. ${ }^{1,2}$

From a clinical standpoint, most

a. Division of Rheumatology. Hospital de Niños "Ricardo Gutiérrez". Argentina

b. Nemours/Alfred I. duPont Hospital for Children. Delaware, USA.

E-mail adress:

Silvia Mónica Meiorin, M.D.: smeiorin@ hotmail.com

Conflict of interest:

None.

Received: 01-21-2013 Accepted: 02-01-2013 innate immunity allowed to recognize the difference between autoimmune and autoinflammatory diseases. From a pathogenic standpoint, both types of diseases are characterized by the chronic activation of the immune system, that eventually leads to tissue inflammation in a genetically predisposed host. However, damage effectors are different in both groups of diseases: in autoinflammatory diseases, the innate immune system causes inflammation, while in autoimmune diseases, it activates the adaptive system which, in turn, is responsible for inflammation..$^{3-5}$

\section{Inflammasome}

Pathogens and warning signals are recognized by pattern receptors (TLR-NLR), which activate different signaling cascades that result in the inflammatory response. One of these receptors, NALP3, is particularly interesting because it serves as the central scaffold of a complex protein called inflammasome. The inflammasome is a molecular platform responsible for the activation of proinflammatory cytokines IL- $1 \mathrm{~b}$ and IL-18. The activation of the NALP3 protein by different agonists unfolds the NALP3 molecule enabling the assembly of other inflammasome components (cardinal, ASC and procaspase 1). Inflammasome oligomerization induces the cleavage and activation of procaspase 1 which promotes the generation of active IL-1b from its inactive precursor, pro-IL1b. This cytokine is secreted from the cytoplasm and triggers an inflammatory response, which includes the production of acute phase reactants by the liver, induces fever (through the hypothalamic thermoregulatory center), stimulates lymphocyte and neutrophil activation, and also acts on bones through 
cartilage resorption and damage stimulation. When inflammasome regulating proteins are mutated, they alter its normal functioning leading to an abnormal hyperinflammation state. ${ }^{6-8}$

New dysfunctional genes and proteins have been identified, and therefore the spectrum and knowledge and recognition of autoinflammatory syndromes are always growing (Table 1).

\section{Hereditary Periodic Fever Syndromes Familial mediterranean fever}

This is the most common autoinflammatory disease, an inherited autosomal recessive disorder related to mutations in the MEFV gene (Mediterranean fever). This gene codes the pyrin protein, which is responsible for regulating the inflammasome. The presence of this mutated protein would favor the assembly of the inflammasome and allow the massive secretion of IL-1b. ${ }^{8}$ This disease has a typical ethnic distribution and is predominant among Turks, non-Ashkenazi Jews, Arabs and Armenians, and also in North Africa and eastern Mediterranean populations. Among Turks and non-Ashkenazi
Jews, the rate of mutation carriers is very high, and ranges from $1: 3$ to $1: 6 .^{1,2}$

In more than $60 \%$ of cases, the disease starts before 5 years old, and when patients are 20 years old, $90 \%$ of them have already developed clinical manifestations. ${ }^{9}$ Fever episodes are short (1-3 days), they are almost always associated with peritoneal or pleural pain due to the occurrence of serositis. Asymmetrical and non-destructive oligoarthritis occurs in $75 \%$ of patients, while an erysipela-like erythema on the ankle or dorsum of the foot is an uncommon finding, but highly suggestive of this disease. Myalgia may appear during a fever episode, and it rarely lasts up to 6 weeks. Neurological manifestations are uncommon in childhood. ${ }^{1,2,9}$ A higher rate of vasculitis (Henoch-Schönlein purpura, nodose panarteritis, and Behçet's disease) has been reported, in addition to IgA nephropathy and diffuse proliferative glomerulonephritis. ${ }^{10}$ During fever episodes, neutrophilia and elevation of acute phase reactants are observed.

The most serious long-term complication is amyloidosis, being proteinuria the most common

TABLE 1. Autoinflammatory diseases. Classification, genes and transmission pattern

\begin{tabular}{|c|c|c|c|c|}
\hline & Disease & $\begin{array}{c}\text { Gene } \\
\text { (chromosome) }\end{array}$ & Protein & Transmission \\
\hline \multirow[t]{3}{*}{$\begin{array}{l}\text { Periodic fever } \\
\text { syndrome }\end{array}$} & $\begin{array}{l}\text { Familial mediterranean } \\
\text { fever }\end{array}$ & $\begin{array}{c}\text { MEFV } \\
16 \mathrm{p} 13.3\end{array}$ & Pyrin & \\
\hline & $\begin{array}{c}\text { Mevalonate-kinase } \\
\text { deficiency }\end{array}$ & $\begin{array}{l}\text { MVK } \\
12 \mathrm{~g} 24\end{array}$ & $\begin{array}{l}\text { Mevalonate- } \\
\text { kinase }\end{array}$ & Autosomal recessive \\
\hline & $\begin{array}{l}\text { TNF receptor associated } \\
\text { periodic syndrome }\end{array}$ & $\begin{array}{l}\text { TNFRSF1A } \\
12 \mathrm{p} 13\end{array}$ & TNF receptor p55 & Autosomal dominant \\
\hline Cryopyrin disorders & $\begin{array}{c}\text { Familial cold } \\
\text { autoinflammatory } \\
\text { syndrome. } \\
\text { Muckle-Wells syndrome } \\
\text { Neonatal onset } \\
\text { multisystem } \\
\text { inflammatory disease }\end{array}$ & $\begin{array}{l}\text { CIAS1 } \\
1 \mathrm{q} 44\end{array}$ & Cryopyrin & Autosomal dominant \\
\hline $\begin{array}{l}\text { Granulomatous } \\
\text { disorders }\end{array}$ & Blau syndrome & $\begin{array}{c}\text { CARD15/NOD2 } \\
16 q 12\end{array}$ & NOD2 & Autosomal dominant \\
\hline \multirow[t]{2}{*}{ Pyogenic disorderss } & PAPA syndrome & $\begin{array}{c}\text { PSTPIP1 } \\
\text { 15q24-q25.1 }\end{array}$ & PSTPIP1 & Autosomal dominant \\
\hline & $\begin{array}{l}\text { Deficiency of } \\
\text { interleukin-1 receptor } \\
\text { antagonist syndrome }\end{array}$ & $\begin{array}{c}\text { IL1RN } \\
2 q\end{array}$ & $\begin{array}{l}\text { IL-1 receptor } \\
\text { antagonist }\end{array}$ & Autosomal recessive \\
\hline
\end{tabular}

PAPA: pyogenic arthritis, pyoderma gangrenosum and acne. 
manifestation and, eventually, kidney failure, which occurs after a variable period of the course of the disease.

Most mutations are localized in the exon $10,{ }^{11}$ particularly founder molecular alterations, such as V726A, M694V, M694I, and M608I. The presence of the M694V mutation has been associated with a more serious condition, especially the development of amyloidosis; however, such association has not been found in all populations. ${ }^{12}$

The treatment of choice is colchicine ${ }^{13}$ in doses of $1 \mathrm{mg}$ /day in adults, up to $2 \mathrm{mg} /$ day in nonresponders. In children younger than 5 years old, the initial dose is $<0.5 \mathrm{mg} /$ day.

Colchicine significantly reduces the incidence of amyloidosis and prevents recurrent episodes of the disease. In the pre-colchicine era, amyloidosis had a prevalence of $60-80 \% .{ }^{1}$ Two thirds of patients have a complete remission of fever episodes, while $20-30 \%$ have a significant improvement with a reduction of fever frequency and seriousness. A small percentage (5-10\%) does not respond to medication, mainly because of their low treatment adherence. Biological treatments, TNF-a blockers and, especially IL-1 inhibitors, are an alternative for patients refractory to or intolerant of colchicine, and also in the case of complications (amyloidosis) or in association with vasculitis. ${ }^{14,18}$

\section{Periodic fever associated with} mevalonate-kinase deficiency

This is also known as hyper-IgD syndrome because it was initially associated with the development of this clinical manifestation. It is predominant in Northern Europe populations, mainly from the Netherlands and France. It is an inherited autosomal recessive disorder related to mutations in the gene that codes the mevalonatekinase enzyme, essential for the biosynthetic pathway of cholesterol and isoprenoid which participate in different cellular processes. ${ }^{1,2}$ Its reduced activity, and the resulting deficiency of this pathway's end factors, would cause an increase in IL-1b secretion. ${ }^{19}$ This disease does not constitute an inflammasome disease per se because the mevalonate-kinase enzyme is not part of this structure.

This condition usually starts in the first year of life, and events are usually triggered by immunizations. The onset of fever is sudden, it lasts for 4 to 6 days and is accompanied by irritability, abdominal pain, vomiting and diarrhea. Lymphadenopathy and splenomegaly are commonly reported. Mucocutaneous manifestations are frequent and include erythematous macules, urticarial lesions, and oral sores. Arthralgias are usually more predominant than symmetrical arthritis. Breakthroughs occur approximately every two months..$^{20-21}$ Symptoms generally wane with growth, but they may continue in adulthood. Amyloidosis has been rarely described as a complication. ${ }^{22,23}$ Neutrophilia and an increase in inflammatory parameters are observed during episodes. High serum IgD values $(>100 \mathrm{IU} / \mathrm{ml})$, both at baseline and during episodes, used to be considered disease markers. ${ }^{20} \mathrm{~A}$ concomitant elevation of IgA has also been reported. Mevalonic acid urinary excretion increases during fever episodes.

The most common MVK gene mutation is the V377I variant, usually associated with a mild phenotype.

Febrile attacks typically show a remarkable response to a short course of corticosteroids (methylprednisolone $1 \mathrm{mg} / \mathrm{kg}$ / day); however, given the high frequency of episodes, some patients require continuous therapy. Under these circumstances, the use of biological drugs (etanercept, IL-1 inhibitors) has allowed to manage febrile attacks recurrence according to certain anecdotal reports. ${ }^{14,21,24,27}$ Other treatments, such as thalidomide, colchicine and simvastatin, were not effective. ${ }^{28,29}$

TNF receptor associated periodic syndrome (TRAPS)

This is a inherited autosomal dominant disorder caused by mutations in the TNF receptor, coded by the TNFRSF1A gene. This disease mainly affects people from Northern Europe, but it has also been described in almost all ethnic groups. ${ }^{1}$ Half of the mutations related to this disorder lead to aminoacid substitutions in cysteine-rich domains of a mature TNF receptor resulting in changes in its tertiary structure. These mutations have higher penetrance with a more severe disease course and phenotype. On the contrary, mutations like R92Q and P46L are associated with a lower penetrance and a milder course. . $^{1,30}$

In normal conditions, TNF receptor activation by TNF leads to the activation of a protease that favors the shedding of the receptor from the cell surface. This process produces a reduction in TNF cell signaling, and the shed receptor is able to bind a free TNF and limit the inflammatory response. Patients affected by this syndrome 
have a defect in receptor shedding that results in a continuous TNF signaling, leading to an inflammatory response. ${ }^{30,31}$

Other mechanisms involved indicate that the mutated receptor is retained in the endoplasmic reticulum instead of having a normal traffic towards the cell surface, where it may activate stimulating signals or induce intracellular stress and probably activate the inflammasome. All these mechanisms lead to a hyperinflammatory state typical in TRAPS patients. ${ }^{30,31}$

It usually begins in childhood, with prolonged fever for 1-3 weeks, and variable symptomfree intervals. Abdominal and chest pain are common due to serous inflammation, together with migrating macular rash that involves the chest or limbs. These lesions are painful and hot, and when they involve the limbs, they are associated with myalgias due to the development of an underlying monocytic fasciitis. Annular plaques or patches can also be observed. The eyes are affected by periorbital edema and conjunctivitis. Arthralgia and arthritis usually involve large joints. Febrile attacks are associated with elevated inflammatory parameters, including serum amyloid A (SAA) level. SAA and PCR persistent elevations during symptom-free intervals are indicative of a sub-chronic disease. Renal amyloidosis is the most serious, long-term complication (with a prevalence of $14-25 \%$ ). ${ }^{1,2}$

Febrile episodes successfully respond to methylprednisolone treatment, but prolonged attacks and the chronic course of this condition make patients become steroid-dependent. Associated toxicity limits their use; in these cases, the use of biological agents has been reported with varied responses. ${ }^{14,33,35}$ While etanercept has been useful for preventing breakthroughs and for treating amyloidosis in some patients, ${ }^{33}$ monoclonal anti-TNF antibodies (adalimumab, infliximab) have been associated with an exacerbation of the disease. ${ }^{14,36}$ For patients in whom anti-TNF treatment fails to control inflammation, other alternative agents such as IL1 blockers have shown an excellent response. ${ }^{14,37,38}$

\section{Cryopyrin disorders}

These are autosomal dominant disorders characterized by different mutations in the CIAS1 gene (also known as NALP-3 or PYPAF1), which codes a protein called cryopyrin. Cryopyrin disorders are grouped in three entities: familial cold autoinflammatory syndrome or familial cold urticaria, Muckle-Wells syndrome, and neonatal onset multisystem inflammatory disease, in ascending order of severity.

Cryopyrin is a member of the NALP cytoplasmic protein sub-family and constitutes the structure of the inflammasome. With certain stimuli, cryopyrin oligomerizes and binds to the ASC adaptor protein. This association is directly activated by the caspase 1 enzyme, which converts pro-IL-1b into its mature form, IL-1b. Thus, the activated inflammasome induces a massive secretion of this pro-inflammatory cytokine and causes hyperinflammation. ${ }^{1,2,8}$

Familial cold urticaria is the mildest cryopyrin disorder, and is characterized by a short duration fever with urticarial exanthema for less than 24 hours induced by exposure to cold. Arthralgia, conjunctivitis, headache, nausea, perspiration and tiredness are common in this condition. ${ }^{39}$

Patients with Muckle-Wells syndrome have recurrent episodes of fever and urticaria since childhood. Its clinical presentation is similar to that of familial urticaria, but it is not strictly triggered by cold. Sensorineural deafness and arthritis usually appear during the course of this condition. ${ }^{39}$

Neonatal onset multisystem inflammatory disease represents the most serious phenotype.

It appears in the first weeks of life, characterized by an urticaria-like rash. Children with this condition have a distinctive and similar facies, characterized by frontal bossing, saddle nose and midface hypoplasia. They develop arthritis and bone overgrowth, especially in the knees and distal limbs (hands and feet). CNS symptoms include aseptic meningitis, cerebral atrophy, increased CSF pressure, sensorineural deafness. chronic papilledema, and visual loss..$^{39,40}$ These patients have a persistent elevation of acute phase reactants, leukocytosis, and anemia.

The key role of cryopyrin in IL-1b secretion suggests a rational criterion for the implementation of anti-IL-1 therapies. The administration of these drugs (anakinra, canakinumab, rilonacept) produces a marked effect in the control of clinical and laboratory inflammatory manifestations. Although their effectiveness is sustained, more serious phenotypes require dose adjustments. ${ }^{14,41,44}$ In certain cases, improvement is achieved, or at least CNS, visual and hearing damage is stabilized. ${ }^{45}$

\section{Pyogenic disorders}

PAPA syndrome

PAPA stands for pyogenic arthritis, pyoderma gangrenosum and acne. The onset of oligoarthritis is usually early in childhood and is characterized 
by recurrent inflammatory episodes similar to a septic arthritis that causes the build-up of pyogenic (neutrophilic) material in the involved joints, destroying the cartilage and synovium. Cultures are sterile. Skin manifestations are also recurrent and episodic, and usually start when the individual is in his/her twenties; they are characterized by skin ulcerations, mainly in the lower limbs, similar to a pyoderma gangrenosum..$^{1,2} \mathrm{Up}$ to this date, three mutations related to this diseased have been identified in the CD2BP1 gene, responsible for synthesizing the CD2BP1 protein. ${ }^{46}$ It has been proposed that the mutated protein has a higher binding affinity for the pyrin protein, thus leading to an increased susceptibility to inflammation. Clinical symptoms tend to respond to oral corticosteroids; however, corticosteroid-refractory patients have shown improvement with anti-IL-1 and TNFblockers. ${ }^{14,46,48}$ The PAPA syndrome is the most difficult autoinflammatory disease to manage from a clinical standpoint.

\section{Deficiency of the interleukin-1-receptor antagonist}

This is a recently-identified autosomal recessive syndrome caused by IL-1 receptor antagonist deficiency, and it starts in the neonatal period with multifocal osteomyelitis, periostitis and pustulosis. Acute phase reactant elevation is observed since birth. Patients have shown homozygous mutations in the IL1RN gene. As a result of these mutations, there is no secretion of IL-1 receptor antagonist, which usually inhibits the proinflammatory action of IL-1. These patients have an excellent response to the replacement therapy with anakinra (recombinant IL-1 receptor antagonist). ${ }^{14,49,50}$

\section{Granulomatous disorders \\ Blau syndrome}

Familial juvenile systemic granulomatosis or Blau syndrome is an autosomal dominant disease characterized by the formation of noncaseating granulomas that impact the joints, skin and uvea. The responsible gene, NOD2 (CARD15), codes a NACHT domain-containing protein. NOD2 is a member of the NOD receptor superfamily, which are bacterial intracellular peptidoglycan receptors. Following stimulation, NOD2 may induce NF$\mathrm{Kb}$ activation and caspase-1-dependant IL- $1 \mathrm{~b}$ secretion. ${ }^{51,52}$ In patients with Blau syndrome, this mutation may cause a gain of the protein function, resulting in a sustained proinflammatory state. This condition usually starts in the first years of life, with symmetrical polyarticular arthritis, which is exuberant and predominantly affects the ankles and the small joints in the hand. A yellowish-brown-color and (ichthyosiform) scaling papular rash has been observed in almost $90 \%$ of patients. Eye involvement is characterized by intermediate uveitis or panuveitis, and 50\% of the patients have complications, including cataracts and secondary glaucoma. Treatment is based on corticosteroids and immunosupressors (methotrexate, ciclosporin A), with varied results. Some reports have suggested a remarkable benefit with infliximab and anti-IL-1 drugs. ${ }^{14,53}$

\section{Molecular analysis}

The discovery of new genes and the identification of dysfunctional proteins related to autoinflammatory syndromes have promoted the development of genetic studies that have led to early diagnoses and new therapies (i.e., IL1-blockers), which have been effective for the prevention of complications, including renal amyloidosis.

However, the genes identified to date are only the tip of the iceberg and account for a small percentage of patients with a geneticallyconfirmed autoinflammatory disease (11-25\%). The main indication for genetic testing is the presence of a clinical pattern compatible with one or more of the syndromes mentioned here. It is not uncommon that with overlapping symptoms or with partial or atypical clinical forms that prevent a precise clinical diagnosis, the testing of disease responsible genes help to make a diagnosis. Flow charts have been developed as a guidance or screening tool to know which genes to test ${ }^{54}$ but testing is not recommended if there are no highly indicative symptoms, because their interpretation may not be conclusive or mutations with incomplete penetrance may be found, which usually do not require treatment.

A basic diagnostic evaluation should include clearly pathogenic variants which are commonly identified in patients with these diseases. Testing usually focuses on the hot spots of each gene (e.g., exon 2-4 of the TNFRSF1A gene in the case of TRAPS, or exon 3 of the NLRP3 gene in the case of cryopyrin disorders).

Genetic interpretation is problematic, so recommendations have been made for testing and interpreting gene variants for the genetic diagnosis of these diseases in order to improve molecular analysis quality and promote lab reporting harmonization and standardization. ${ }^{55,56}$ 


\section{Registries}

The low prevalence of these diseases, their phenotype variability and the fact that clinical experience is fragmented among a few health facilities in different countries have been the main limitations for the study of these entities. As a counteracting measure, several international studies or registries (Eurofever, Infevers, Blau Registry) have been developed to expand the knowledge regarding their clinical presentation, complications and management, to streamline and quickly recognize them in the medical community, and also to provide information to parents and patients. ${ }^{57,59}$

\section{REFERENCES}

1. Federici S, Caorsi R, Gattorno M. The autoinflammatory diseases. Swiss Med Wkly 2012, 19;142w13602.

2. Gattorno M, Federici S, Pelagatti MA, Caorsi R, et al. Diagnosis and management of autoinflammatory diseases in childhood. J Clin Immunol 2008;28(Suppl 1):S73-83.

3. Doria A, Dayer JM, Punzi L. Autoinflammatory diseases: How to put the fire inside the body out?. Autoimmun Rev 2012;12(1):1-4.

4. Doria A, Zen M, Bettio S, Gatto M, et al. Autoinflammation and autoimmunity: Bridging the divide. Autoimmun Rev 2012;12(1):22-30.

5. Mills KH, Dunne A. Immune modulation: IL-1, master mediator or initiator of inflammation. Nat Med 2009;15(12):1363-4.

6. Church LD, Cook GP, McDermott MF. Primer: inflammasomes and interleukin 1beta in inflammatory disorders. Nat Clin Pract Rheumatol 2008;4(1):34-42.

7. Lachmann HJ, Quartier P, So A, Hawkins PN. The emerging role of interleukin-1 b in autoinflammatory diseases. Arthritis Rheum 2011;63(2):314-24.

8. Ozkurede VU, Franchi L. Immunology in clinic review se $\neg$ ries; focus onautoinflammatory diseases: role of inflammasomes in autoinflammatory syndromes. Clin Exp Immunol 2012;167(3):382-90.

9. Drenth J, van der Meer J. Hereditary Periodic Fever. $N$ Engl J Med 2001;345(24):1748-57.

10. Samuels J, Ozen S. Familial Mediterranean Fever and the other autoinflammatory syndromes: evaluation of the patient with recurrent fever. Curr Opin Rheumatol 2006;18(1):108-17.

11. Soriano A, Manna R. Familial Mediterranean fever: New phenotypes. Autoimmun Rev 2012;12(1):31-7.

12. Lidar M, Yonath $\mathrm{H}$, Shechter $\mathrm{N}$, Sikron $\mathrm{F}$, et al. Incomplete responsetocolchicineinM694VhomozygoteFMFpatients. Autoimmun Rev 2012;12(1):72-6.

13. Goldfinger SE. Colchicine for Familial Mediterranean Fever. N Engl J Med 1972;287(25):1302.

14. Ter Haar N, Lachmann H, Ozen S, Woo P, et al. Treatment of autoinflammatory diseases: results from the Eurofever Registry and a literature review. Ann Rheum Dis 2012 Jun 29.

15. Caorsi R, Federici S, Gattorno M. Biologic drugs in autoinflammatory syndromes. Autoimmun Rev 2012;12(1):81-6.

16. Gattorno M, Martini A. Treatment of autoinflammatory syndromes. Curr Opin Pediatr 2010;22(6):771-8.

17. Meinzer U, Quartier P, Alexandra JF, Hentgen $\mathrm{V}$, et al. Interleukin-1 targeting drugs in familial. Mediterraneanfever: a case series and a review of the literature. Semin Arthritis Rheum 2011;41(2):265-71.
18. Ozgocmen S, Akgul O. Anti-TNF agents in familial Mediterranean fever: report of three cases and review of the literature. Mod Rheumatol 2011;21(6):684-90.

19. Normand S, Massonnet B, Delwail A, Favot L, et al. Specific increase in caspase-1 activity and secretion of IL-1 family cytokines: a putative link between mevalonate kinase deficiency and inflammation. Eur Cytokine Netw 2009;20(3):101-7.

20. van der Hilst JC, Bodar EJ, Barron KS, Frenkel J, et al. Longterm follow-up, clinical features, and quality of life in a series of 103 patients with hyperimmunoglobulinemia D syndrome. Medicine (Baltimore) 2008;87(6):301-10.

21. Bader-Meunier B, Florkin B, Sibilia J, Acquaviva C, et al. Mevalonate kinase deficiency: a survey of 50 patients. Pediatrics 2011;128(1):e152-9.

22. Obici L, Manno C, Muda AO, Picco P, et al. First report of systemic reactive (AA) amyloidosis in a patient with the hyperimmunoglobulinemia $\mathrm{D}$ with periodic fever syndrome. Arthritis Rheum 2004;50(9):2966-9.

23. LachmannHJ, GoodmanHJ,AndrewsPA, Gallagher H, etal. AAamyloidosiscomplicatinghyperimmunoglobu $\neg$ linemia D with periodic fever syndrome: a report of two cases. Arthritis Rheum 2006;54(6):2010-4.

24. Korppi M, Van Gijn ME, Antila K. Hyperimmunoglobulinemia D and periodic fever syndrome in children. Review on therapy with biological drugs and case report. Acta Paediatr 2011;100(1):21-5.

25. Topalo lu R, Ayaz NA, Waterham HR, Yüce A, et al. Hy $\neg$ perimmunoglobulinemia D and periodic fever syndrome; treatment with etanercept and follow-up. Clin Rheumatol 2008;27(10):1317-20.

26. Takada K, Aksentijevich I, Mahadevan V, Dean JA, et al. Favorable preliminary experience with etanercept in two patients with the hyperimmunoglobulinemia Dand periodic fever syndrome. Arthritis Rheum 2003;48(9):2645-51.

27. Demirkaya E, Caglar MK, Waterham HR, Topalo lu R, Ozen S. A patient with hyper-IgD syndrome responding to anti-TNF treatment. Clin Rheumatol 2007;26(10):1757-9.

28. Simon A, Drewe E, van der Meer JW, Powell RJ, et al. Simvastatin treatment for inflammatory attacks of the hyperimmunoglobulinemiaDandperiodicfeversyndrome. Clin Pharmacol Ther 2004;75(5):476-83.

29. Drenth JP, Vonk AG, Simon A, Powell R, van der Meer JW. Limited efficacy of thalidomide in the treatment of febrile attacks of the hyper-IgD and periodic fever syndrome: a randomized, double-blind, placebo-controlled trial. J Pharmacol Exp Ther 2001;298(3):1221-6.

30. Cantarini L, Lucherini OM, Muscari I, Frediani B, et al. Tumour necrosis factor receptor-associated periodic syn $\neg$ drome (TRAPS): State of the art and future perspectives. Autoimmun Rev 2012;12(1):38-43.

31. Pettersson T, Kantonen J, Matikainen S, Repo H. Setting up TRAPS. Ann Med 2012;44(2):109-18.

32. Hull KM, Drewe E, Aksentijevich I, Singh HK, et al. The TNF receptor-associated periodic syndrome (TRAPS): emerging concepts of an autoinflammatory disorder. Medicine (Baltimore) 2002;81(5):349-68.

33. Drewe E, McDermott EM, Powell PT, Isaacs JD, Powell RJ. Prospective study of anti-tumour necrosis factor receptor superfamily 1B fusion protein, and case study of anti-tumour necrosis factor receptor superfamily $1 \mathrm{~A} \mathrm{fu}$ sion protein, in tumour necrosis factor receptor associated periodic syndrome (TRAPS): clinical and laboratory findings in a series of seven patients. Rheumatology (Oxford) 2003;42(2):235-9.

34. Bulua AC, MogulDB, Aksentijevich I, Singh H, etal. Efficacy of etanercept in the tumor necrosis factor receptorassociated periodic syndrome: a prospective, open-label, dose- 
escalation study. Arthritis Rheum 2012;64(3):908-13.

35. DreweE,Huggins ML,MorganAG,Cassidy MJ,Pow 7 ell RJ. Treatment of renal amyloidosis with etanercept in tumour necrosis factor receptor-associated periodic syndrome. Rheumatology (Oxford) 2004;43(11):1405-8.

36. Jacobelli S, André M, Alexandra JF, Dodé C, Papo T. Failure of anti-TNF therapy in TNF Receptor 1-Associated Periodic Syndrome (TRAPS). Rheumatology (Oxford) 2007;46(7):1211-2.

37. Gattorno M,PelagattiMA, Meini A, Obici L, etal. Persistent efficacy of anakinra in patients with tumor necrosis factor receptor-associated periodic syndrome. Arthritis Rheum 2008;58(5):1516-20.

38. Obici L, Meini A,Cattalini M,ChiccaS, etal. Favourable and sustained response to anakinra in tumour necrosis fac $\neg$ tor receptor-associated periodic syndrome (TRAPS) with or withoutAAamyloidosis. Ann Rheum Dis 2011;70(8):1511-2.

39. Neven B, Prieur AM, Quartier dit Maire P. Cryopyrinopa thies: update on pathogenesis and treatment. Nat Clin Pract Rheumatol 2008;4(9):481-9.

40. Goldbach-Mansky R. Current status of understanding the pathogenesis and management of patients with NOMID / CINCA. Curr Rheumatol Rep 2011;13(2):123-31.

41. Goldbach-Mansky R, Dailey NJ, Canna SW, Gelabert A, et al. Neonatal-onset multisystem inflammatory disease responsive to interleukin-1beta inhibition. $N$ Engl J Med 2006;355(6):581-92.

42. Hoffman HM, Throne ML, Amar NJ, Cartwright RC, et al. Long-term efficacy and safety profile of rilonacept in the treatment of cryopryin-associated periodic syndromes: results of a 72-week open-label extension study. Clin Ther 2012;34(10):2091-103.

43. Koné-Paut I, Lachmann HJ, Kuemmerle-Deschner JB, Hachulla E, et al. Sustained remission of symptoms and improved health-related quality of life in patients with cryopyrin-associated periodic syndrome treated with canakinumab: results of a double-blind placebocontrolled randomized withdrawal study. Arthritis Res Ther 2011;13(6):R202.

44. Koné-Paut I, Piram M. Targeting interleukin-1 b in CAPS (cryopyrin-associated periodic) syndromes: what did we learn? Autoimmun Rev 2012;12(1):77-80.

45. Sibley CH, Plass N, Snow J, Wiggs EA, et al. Sustained response and prevention of damage progression in patients with neonatal-onset multisystem inflammatory disease treated with anakinra: a cohort study to determine three- and five-year outcomes. Arthritis Rheum 2012;64(7):2375-86.
46. Demidowich AP, Freeman AF, Kuhns DB, Aksentijevich I, et al. Brief report: genotype, phenotype, and clinical course in five patients with PAPA syndrome (pyogenic sterile arthritis, pyoderma gangrenosum, and acne). ArthritisRheum 2012;64(6):2022-7.

47. LeeH,ParkSH,KimSK, ChoeJY,ParkJS. Pyogenic arthritis, pyoderma gangrenosum, and acne syndrome (PAPA syndrome) with E250K mutation in CD2BP1 gene treated with the tumor necrosis factor inhibitor adalimumab. Clin Exp Rheumatol 2012;30(3):452.

48. Tofteland ND, Shaver TS. Clinical efficacy of etanercept for treatment of PAPA syndrome. J Clin Rheumatol 2010;16(5):244-5.

49. Aksentijevich I, Masters SL, Ferguson PJ, Dancey P, et al. An autoinflammatorydiseasewithdeficiencyoftheinterleuk in1-receptor antagonist. N Engl J Med 2009;360(23):2426-37.

50. Jesus AA, Osman M, Silva CA, Kim PW, et al. A novel mutation of IL1RN in the deficiency of interleukin-1 receptor antagonist syndrome: description of two unrelated cases from Brazil. Arthritis Rheum 2011;63(12):4007-17.

51. RoseCD,Martin TM, Wouters CH. Blau syndrome revisited. Curr Opin Rheumatol 2011;23(5):411-8.

52. Sfriso P, Caso F, Tognon S, Galozzi P, et al. Blau syndrome, clinical and genetic aspects. Autoimmun Rev 2012;12(1):4451.

53. Aróstegui JI, Arnal C, Merino R, Modesto C, et al. NOD2 gene-associated pediatric granulomatous arthritis: clini $\neg$ cal diversity, novel and recurrent mutations, and evidence of clinical improvement with interleukin-1 blockade in a Spanish cohort. Arthritis Rheum 2007;56(11):3805-13.

54. Gattorno M, Sormani MP, D'Osualdo A, Pelagatti MA, et al. A diagnostic score for molecular analysis of hereditary autoinflammatory syndromes with periodic fever in children. Arthritis Rheum 2008;58(6):1823-32.

55. Shinar Y, Obici L, Aksentijevich I, Bennetts B, et al. Guidelines for the genetic diagnosis of hereditary recurrent fevers. Ann Rheum Dis 2012;71(10):1599-605.

56. Touitou I. New genetic interpretation of old diseases. Autoimmun Rev 2012;12(1):5-9.

57. Toplak N, Frenkel J, Ozen S, Lachmann HJ, et al. An international registry on autoinflammatory diseases: the Eurofever experience. Ann Rheum Dis 2012;71(7):1177-82.

58. Sarrauste de Menthière $C$, Terrière $S$, Pugnère $D$, Ruiz $M$, et al. INFEVERS: the Registry for FMF and hereditary inflammatory disorders mutations. Nucleic Acids Res 2002;31(1):282-5.

59. RoséCD, Wouters $\mathrm{CH}$, Meiorin S, Doyle TM, etal. Pediatric granulomatous arthritis: an international registry. Arthritis Rheum 2006;54(10):3337-44. 